\title{
Thermal Transients and Convective Particle Motion in Dense Granular Materials
}

\author{
P. Rognon and I. Einav* \\ School of Civil Engineering, J05, The University of Sydney, Sydney, New South Wales 2006, Australia
}

(Received 3 June 2010; published 17 November 2010)

\begin{abstract}
The mechanism of dry granular convection within dense granular flows is mostly neglected by current analytical heat equations describing such materials, for example, in geophysical analyses of shear gouge layers of earthquake and landslide rupture planes. In dry granular materials, the common assumption is that conduction by contact overtakes any other mode of heat transfer. Conversely, we discover that transient correlated motion of heated grains can result in a convective heat flux normal to the shear direction up to 3-4 orders magnitude larger than by contact conduction. Such a thermal efficiency, much higher than that of water, is appealing and might be common to other microscopically structured fluids such as granular pastes, emulsions, and living cells.
\end{abstract}

The temperature evolution within sheared granular layers is balanced by heat production and transfer. Identifying the dominant modes of the heat transfer is therefore critical to temperature predictions. As typical with any classical fluid, the two most important modes of heat transfer during granular flow are those by conduction and convection. Heat conduction is now well understood to rely on the properties controlling the contact network: contact size, anisotropy, and heterogeneity [1-4]. Various studies examined the effect of convection on the heat transfer between the flowing granular materials and bounding walls [5]. In such cases where the wall interacts with the flow, the heat transfer is affected by the first layer of wall-contacting grains that may or may not recirculate. Moreover, the efficiency of the overall heat transfer depends on the thermal properties of the wall. Here, we examine the heat transfer that is purely intrinsic to the granular media, independent of wall properties. Intrinsic to the flow, the heat is carried by grains as they move. We term this process dry granular convection. Some heat is thus simply flowing along the mass flux. In addition to this obvious advection, in turbulent flow of water vortices develop and induce heat transfer normal to the mass flux [6]. Granular flows exhibit similar turbulent-like patterns of velocity vortices [7], leading to temperature mixing normal to the mass flux [8], but the corresponding heat flux has not been studied. Unlike water, vortice-like motion in granular media results from force chain buckling, leading to high fluctuations in local strain and kinetic energies, and thereafter, as we show, to thermal convection that increases with vertical stresses.

In this Letter, we discover that dry granular convection does add a striking contribution to the overall heat transfer budget in dense granular flows. The study is based on a series of numerical experiments using thermal discrete element method [2]; the motion and the temperature evolution of the grains are simulated while they experience a plane shear flow and a temperature gradient in the direction transverse to the shear plane [Fig. 1(a)]. In the following, we will first introduce the physical properties and the associated dimensionless numbers describing the system. The measurement of convection and conduction fluxes for various loading and grain properties will then be presented. Finally, we will prove that the convective flux comes directly from the correlation between velocity and temperature fluctuations; it is therefore driven by the temperature mixing due to transient vortex motions, and hindered by the contact conduction bringing the temperature back to thermal equilibrium.

Rational description of heated granular sheared layers.-Practical issues such as earthquakes, landslides, and granular lubrication involve a wide range of grain properties and loading intensities [9]. The set of relevant dimensionless numbers describing the physical properties of the grains is then given by their typical diameter $d$, mass $m$, mass density $\rho$, Young's modulus $E[\mathrm{~Pa}]$, bulk conductivity $k_{s}\left[\mathrm{~W} \mathrm{~m}^{-1} \mathrm{~K}^{-1}\right]$, and bulk heat capacity $c$ $\left[\mathrm{J} \mathrm{kg}^{-1} \mathrm{~K}^{-1}\right]$. The loading conditions can generally be described by a normal stress $\sigma_{y y}$ and a shear rate $\dot{\gamma}$. It is then possible to detect four fundamental time scales: the shear time $t_{\dot{\gamma}}=\dot{\gamma}^{-1}$, the inertial time $t_{i}=\sqrt{\frac{m}{d \sigma_{y y}}}$, the collision time $t_{c}=\sqrt{\frac{m}{E d}}$, and the thermal time $t_{\mathrm{th}}=\frac{m c}{d k_{\mathrm{s}}}$. Therefore, only three independent dimensionless numbers may describe our system. Two of them have been broadly used to express constitutive laws for granular flow: the inertial number $I=t_{i} / t_{\dot{\gamma}}$ describing the shear state, and the stiffness number $\kappa=\left(t_{c} / t_{i}\right)^{2}=\sigma_{y y} / E$ reflecting the degree of deformation sustained by the grains $[4,10]$. We introduce a third dimensionless number, which we term the thermal number, defined as $\tau=t_{\text {th }} / t_{i}$, among other possible combinations. The possible range of values these three numbers can attain concerning various practical problems is discussed in Table I.

Thermomechanics of grain contacts.-We consider a model material whose grains are spherical and slightly polydispersed $(d \pm 20 \%)$. The grains interact by direct 

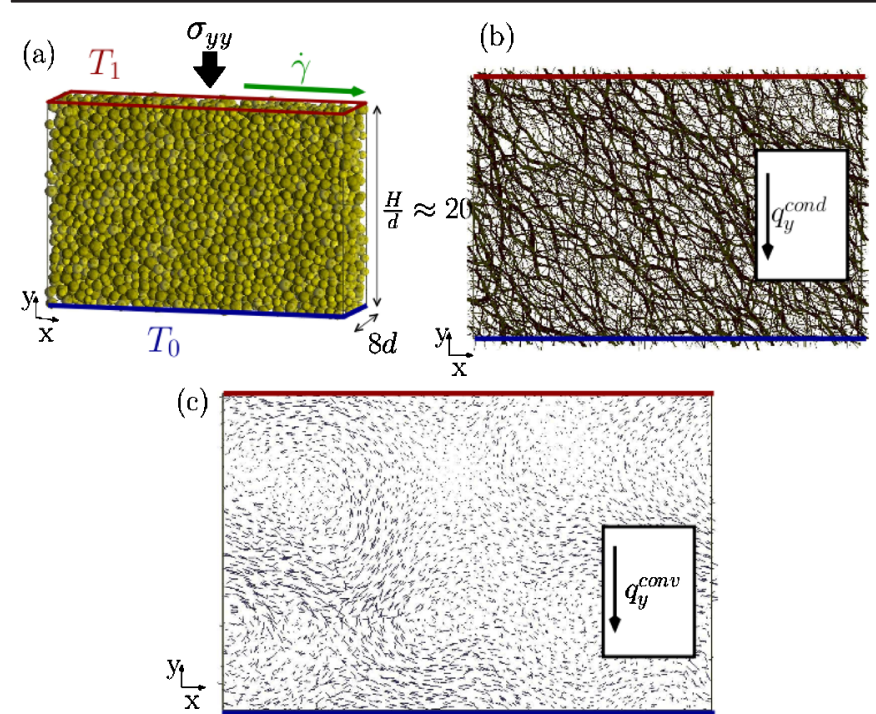

FIG. 1 (color online). Two modes of heat transfer within a sheared granular layer: contact conduction along the contact network and dry granular convection by correlated grain motion. (a) 5000 spheres in a three-dimensional periodic domain are subjected to a constant shear rate $\dot{\gamma}$ and a constant normal stress $\sigma_{y y}$; the top boundary is hot, and the bottom is cold, so that the global thermal gradient along $y, \nabla T_{0}=\frac{T_{1}-T_{0}}{H}$, is constant with time (the grains moving out of the bottom return back from the top with a temperature rise of $\nabla T_{0} H$, and vice versa). (b) The contact network controls the conduction heat flux density along $y, q_{y}^{\text {cond }}$ (each plotted segment connects the centers of two contacting grains and is thickened proportionally to the normal contact force). (c) The velocity fluctuation field controls the convective heat flux density along $y, q_{y}^{\text {conv }}$ (each plotted segment starts from the center of a grain and is lengthened proportionally to its velocity vector minus the laminar velocity field, $\dot{\gamma} y_{i}$, along $x$ ).

contacts which also transmit heat by conduction. The friction coefficient $\mu$ is set to 0.4 and the coefficient of normal restitution to 0.8 . The normal contact force satisfies the Hertz model, $F_{n}=E a^{3} / d$, with $a$ being the contact radius. As expressed in [4], we also include the contact

TABLE I. Wide practical appeal is achieved by changing three dimensionless numbers $\kappa, I$, and $\tau$. Their chosen range of values covers important phenomena [9]: (i) a $10 \mathrm{~km}$ deep earthquake $\left(\sigma_{y y}=10^{8} \mathrm{~Pa}\right)$ involving a $1 \mathrm{~mm}$ width fault gouge, a slip velocity from 0 to $10 \mathrm{~ms}^{-1}$, and $d=100 \mu \mathrm{m}$ grains; (ii) a $10 \mathrm{~m}$ thick landslide $\left(\sigma_{y y}=10^{5} \mathrm{~Pa}\right)$, with deformations localized within a thin $1 \mathrm{~cm}$ basal shear layer, driven by a slip velocity from 0 to $10 \mathrm{~ms}^{-1}$, and $d=100 \mu \mathrm{m}$ grains. In both cases, the bulk density of grains is $3 \times 10^{3} \mathrm{~kg} / \mathrm{m}^{3}$, their Young's modulus is $10^{11} \mathrm{~Pa}$, and their ratio $c / k_{s}$ of heat capacity to bulk heat conductivity ranges from 1 to $10^{3} \mathrm{SI}$, which describes most solids.

\begin{tabular}{lccc}
\hline \hline & $\kappa=\frac{\sigma_{y y}}{E}$ & $I=\dot{\gamma} \sqrt{\frac{m}{\sigma_{y y} d}}$ & $\tau=\sqrt{\frac{m \sigma_{y y}}{d}} \frac{c}{k_{s}}$ \\
\hline Earthquakes & $10^{-3}$ & $0 \rightarrow 10^{-1}$ & $1 \rightarrow 10^{5}$ \\
Landslides & $10^{-6}$ & $0 \rightarrow 10^{-2}$ & $10^{-1} \rightarrow 10^{4}$ \\
Present study & $10^{-3}$ & $10^{-3} \rightarrow 10^{-1}$ & $10^{-2} \rightarrow 10^{5}$ \\
\hline \hline
\end{tabular}

torques for twist and rolling with their respective sliding and rolling criteria. The heat flux $\phi_{i j}[\mathrm{~W}]$ between two contacting grains is described by the model of conductance for perfectly smooth contacts: $\phi_{i j}=-2 k_{s} a\left(T_{j}-T_{i}\right)$. It accounts for the constriction of the heat flux [3,11]: the temperature $T_{i, j}$ is almost homogeneous within grains apart from the small region of typical size $a$ alongside the contact where the temperature gradient is localized. Therefore, the small Hertzian contacts per particle are thermally independent. The transient heat flux of the contacting grains is approximated by the steady-state value. This assumption is discussed in [3,12] and proves to be reasonable even for collision-dominated flows, and even more so in our dense flows with sustained contacts. The thermal discrete element method involves the numerical integration of grain motion by Newton's law, considering the local force and torque balances. In addition, the grain temperatures are updated, given their local heat budget: $\dot{T}_{i}=\frac{1}{c m_{i}} \sum_{j} \phi_{i j}$, which includes the heat flux contribution from all contacting particles, indexed by $j$ [2].

Measuring dry granular convection.-The numerical experiments begin with the generation of a flow by subjecting a random configuration of grains to a steady shear rate and designated normal stress [Fig. 1(a)] [13]. After some time, the flow reaches a steady regime with no shear localization, during which the sample height, the shear stress, and the solid fraction $\nu$ remain constant apart from small fluctuations. The "heat" part of the experiment starts when the steady regime is reached; grain temperatures are initially set according to a constant gradient $\nabla T_{0}$ along $y: T_{i}=\nabla T_{0} y_{i}$. They are then free to evolve, but constrained to a thermal condition where the difference between the temperatures at the top and bottom boundaries remains constant to ensure a constant global temperature gradient of $\nabla T_{0}$. The heat flux densities $\left[\mathrm{W} \mathrm{m}^{-2}\right.$ ] from the contact conduction $\vec{q}^{\text {cond }}$ and from the dry granular convection $\vec{q}^{\text {cond }}$ [Figs. 1(b) and 1(c)] are measured by

$$
\vec{q}^{\text {cond }}=\frac{1}{\Omega} \sum_{c} \phi_{c} \vec{r}_{c}, \quad \vec{q}^{\text {conv }}=\frac{1}{\Omega} \sum_{i} m_{i} c T_{i} \vec{v}_{i} .
$$

The first sum represents the contribution of all contacts transferring a heat flux $\phi$ along the center-to-center vector $\vec{r}$. The second sum runs over all of the grains, each of which moves with a velocity $\vec{v}_{i}$ and carries heat with a value of $m c T$. These two formulations thus denote a spatial average over the entire sample volume $\Omega$, and are used to measure snapshots of the two fluxes at any time during the shear.

Figure 2 shows the individual contribution to the overall budget of heat transfer by the dry granular convection and through the contact conduction. The behavior is similar, almost irrespective of the imposed inertial number: the convection flux increases mostly linearly with the thermal number $\tau$, approximately by $q_{y}^{\text {conv }} \approx 10^{-2} q_{s} \tau$, while the conduction flux is almost constant, $q_{y}^{\text {cond }} \approx 0.1 q_{s}$. For thermal numbers larger than about 10 , the convection becomes the dominant mode of heat transfer. Although not shown 
here, it is well known that the conduction flux depends on the degree of grain deformation, and scales with $q_{s} \kappa^{1 / 3}$ for Hertzian contacts [1-4]. In any case, the conduction flux has a clear upper bound: the heat flux of a continuous solid, $q_{s}=k_{s} \nabla T_{0}$. In contrast, the convective flux is not subjected to such a limitation, and actually becomes larger than $q_{s}$ for $\tau \gtrsim 100$. In classical liquids, the Nusselt number $\mathrm{Nu}=$ $q_{y}^{\text {conv }} / q_{y}^{\text {cond }}$ is often used to describe the relative importance of the convection versus conduction. For water, the maximum values are of the order of 100 [6]. For our granular material, this ratio is proportional to the thermal number and can thus reach values up of $10^{4}$ :

$$
\mathrm{Nu} \approx 0.1 \sqrt{\frac{m \sigma_{y y}}{d}} \frac{c}{k_{s}} .
$$

Origin of convection. - In the current system there is no net mass flux along $y$, which is why the heat convection cannot be associated with some advection along the mean flow velocity. Instead, as shall be demonstrated next, here the measured convection comes directly from both the velocity and temperature fluctuations. For that purpose, we denote the fluctuation parts of the temperatures and velocities from the corresponding mean field by $\tilde{T}$ and $\tilde{v}$. In the current simulations, the temperature and velocity of a given grain can then be expressed by $T_{i}=\nabla T_{0} y_{i}+\tilde{T}$ and

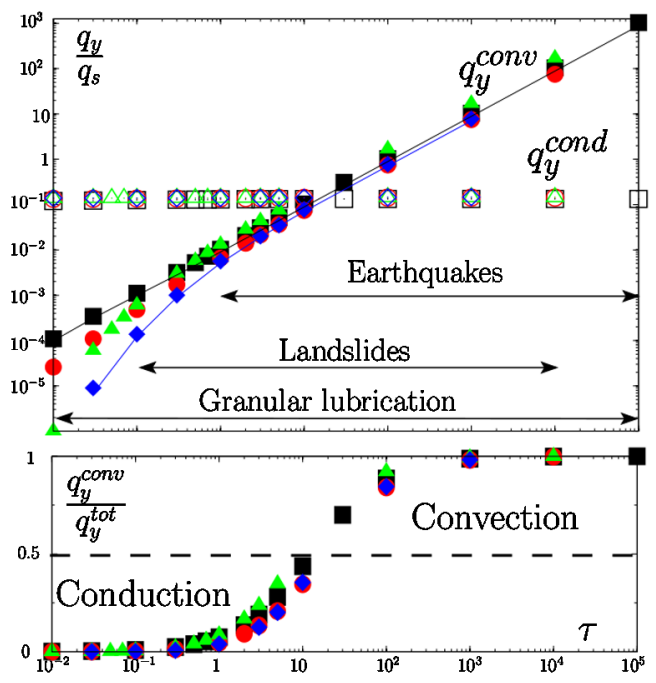

FIG. 2 (color online). Relative contribution of the dry granular convection and contact conduction to the heat transfer as a function of the thermal number $\tau$, for inertial number $I=3 \times$ $10^{-3}(\bullet), 10^{-2}(\boldsymbol{\Delta}), 3 \times 10^{-2}(\bullet)$, and $10^{-1}(\boldsymbol{\square})$. Top panel: Conduction (open symbols) and convection (filled symbols) fluxes in units of $q_{s}=k_{s} \nabla T_{0}$, which would be the conduction flux of the material if it was a continuous solid; the lines correspond to the convection flux obtained by Eq. (3). Bottom panel: The ratio of heat transferred by convection to the total budget $\left(q_{y}^{\text {tot }}=q_{y}^{\text {conv }}+q_{y}^{\text {cond }}\right)$. The points correspond to values averaged both spatially over the whole sample [see Eqs. (1)], and temporally over 100 snapshots taken at every increment of 0.1 shear deformation. By definition, when $\dot{\gamma}=0$ (i.e. no shear) there should be no convection, but notice that the convection rapidly increases with $\tau$ even for the very low $I=3 \times 10^{-3}$. $v_{y, i}=0+\tilde{v}_{i}$, respectively. Therefore, the dry convective flux $q_{y}^{\text {conv }}$ in Eq. (1) can be rewritten as follows: $q_{y}^{\text {conv }}=$ $\frac{1}{\Omega} \sum_{i=1}^{N} m_{i} c_{i}\left(\nabla T_{0} y_{i}+\tilde{T}_{i}\right) \tilde{v}_{i}$. The heat capacity is the same for all grains, and the effect of the grain mass distribution can be safely neglected since our system involves only a minimal polydispersity. Next, if the velocity fluctuation is statistically independent of the $y$ position, we may further assume that the sum of $\nabla T_{0} y \tilde{v}$ would be null. The previous equation thus becomes $q_{y}^{\text {conv }}=\frac{m c N}{\Omega} \frac{1}{N} \sum_{i=1}^{N} \tilde{T}_{i} \tilde{v}_{i}$. The first ratio is equal to $\rho c \nu, \nu$ being the solid fraction of the sample, while the sum is the expected value of the product $T_{i} \tilde{v}_{i}$ measured for the sample of $N$ grains, $\mathbb{E}\left(\tilde{T}_{i} \tilde{v}_{i}\right)$. The expected value of the product can be developed as $\mathbb{E}\left(\tilde{T}_{i} \tilde{v}_{i}\right)=\mathbb{E}\left(\tilde{T}_{i}\right) \mathbb{E}\left(\tilde{v}_{i}\right)+\operatorname{Cov}\left(\tilde{T}_{i} ; \tilde{v}_{i}\right)$. The first product to the right of the equality is null since the expected value of the fluctuations vanishes by definition. The convective flux is then proportional to the covariance of the temperature and velocity fluctuations, $\operatorname{Cov}\left(\tilde{T}_{i} ; \tilde{v}_{i}\right)$ :

$$
q_{y}^{\mathrm{conv}}=\rho c \nu \mathbb{E}\left(\tilde{T}_{i} \tilde{v}_{i}\right)=\rho c \nu \operatorname{Cov}\left(\tilde{T}_{i} ; \tilde{v}_{i}\right) .
$$

Figure 2 shows that this expression exactly matches the convective flux measurements from Eq. (1). This highlights the pivotal role of the correlation of temperature and velocity fluctuations: the convective flux would attain a non-null value only if the two fluctuations are correlated. In other words, here the convection becomes appreciable when grains moving up are more likely to be cooler than the linear temperature profile, while those moving down are more likely to be hotter than it.

Figures 3 and 4 show that such a correlation occurs during transient events of large vortex motions, which induce a rise in the velocity fluctuations, shortly followed

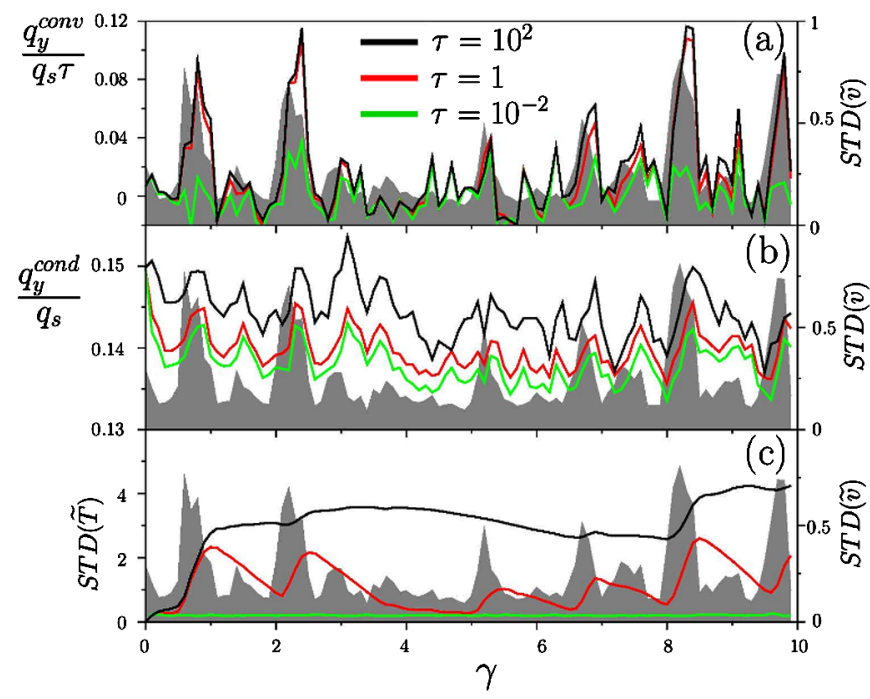

FIG. 3 (color online). Transients of dry granular convection: (a-c) dry granular convection, conduction flux, the standard deviation of the temperature fluctuations $\operatorname{STD}(\tilde{T})$, and of the velocity fluctuations $\operatorname{STD}(\tilde{v})$ (right axis, filled curve), all as a function of the shear deformation $\gamma$, for mechanically identical flows $\left(I=10^{-2}\right)$ with three different thermal numbers $\tau$. 


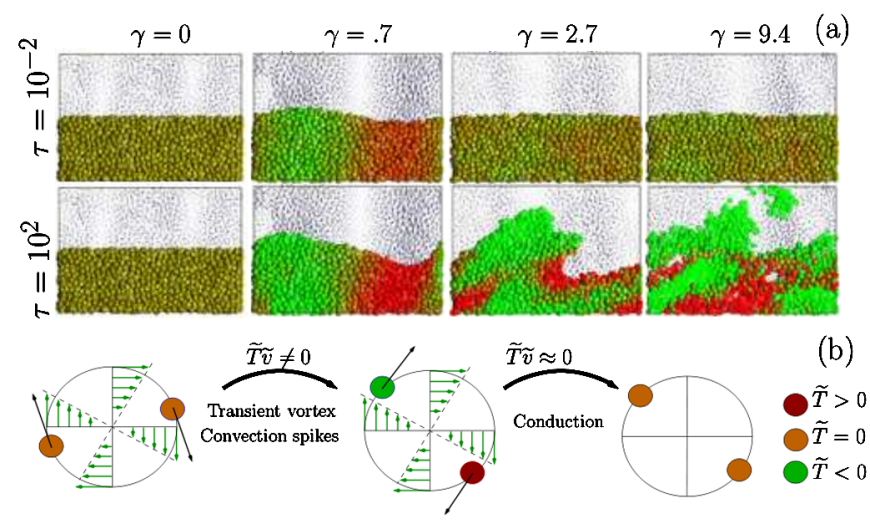

FIG. 4 (color online). Thermal cycles: transient vortex mixing versus conduction. (a) Snapshots of temperature fluctuations along the shear deformation for mechanically identical flows ( $I=10^{-2}$ ) with two different thermal numbers $\tau$ (corresponding to Fig. 3). Only grains cooler than the overall average temperature $\nabla T_{0} H / 2$ are plotted, thereby showing the isothermal line; above this line, the plotted segments represent the velocity field as in Fig. 1(c). Grain colors reflect their temperature fluctuation. (b) Sketch of a thermal cycle with transient vortex mixing and conduction.

by another rise, this time in the temperature fluctuations. This correlation results in high spikes of convection flux, without any visible effect on the conduction flux. The conduction acts against the dry granular convection, by continuously bringing back the grain temperature to the constant gradient equilibrium. However, this process takes time. For small thermal number $(\tau<1)$, the conduction tends to equilibrate the temperature quicker than another vortex may remix it, thereby decorrelating the temperature and velocity fluctuations, and decreasing the convection flux. Conversely, for large thermal number $(\tau>1)$ the conduction acts more slowly than the transients of vortex mixing, which results in large spikes of convection fluxes, scaling with $\tau$.

This Letter motivates further study of the heat transfer problem in dense granular flows (even if dry) as is done in turbulent flows of water: by accounting for the convection. It was shown that dry granular convection may easily overcome the contribution by conduction to the overall heat flux budget, even normal to the net mass flux direction. The magnitude of the convective heat flux, which was neglected so far, should motivate us to reanalyze the thermomechanics of gouge granular materials sheared during earthquakes and landslides. This mode of heat transfer could also be significant for complex fluids with similar microstructures (granular suspensions, foams, emulsions, and living cells) for which large correlated motion has been reported. Further research should be devoted to find appropriate means to predict and characterize the transient nature of the vortices in granular flows, while connecting those transients to the mechanical properties of the grains (their shape, size, and polydispersity) and to the loading conditions.
We thank J. Patterson, E. Veveakis, and J. Bonivin for fruitful discussions. Financial support for this research from the Australian Research Council through Grant No. DP1096958 is gratefully appreciated.

*itai.einav@sydney.edu.au

[1] D. Kunii and J. Smith, AIChE J. 6, 71 (1960); G. Batchelor and R. O'Brien, Proc. R. Soc. A 355, 313 (1977).

[2] W. Vargas and J. McCarthy, AIChE J. 47, 1052 (2001); Chem. Eng. Sci. 57, 3119 (2002).

[3] A. Smart, P. Umbanhowar, and J. Ottino, Europhys. Lett. 79, 24002 (2007).

[4] P. Rognon, I. Einav, J. Bonivin, and T. Miller, Europhys. Lett. 89, 58006 (2010).

[5] J. Patton, R. Sabersky, and C. Brennen, Int. J. Heat Mass Transf. 29, 1263 (1986); B. Chaudhuri, F. Muzzio, and M. Tomassone, Chem. Eng. Sci. 61, 6348 (2006); M. Kwapinska, G. Saage, and E. Tsotsas, Powder Technol. 181, 331 (2008); M.L. Hunt, Annual Review of Heat Transfer 3, 163 (1990); V. Natarajan and M. Hunt, Experimental Heat Transfer 10, 89 (1997).

[6] B. Petukhov and A. Polyakov, Heat Transfer in Turbulent Mixed Convection, edited by B. Launder (Hemisphere, New York, 1988); F. Incropera, D. DeWitt, T. Bergman, and A. Lavine, Fundamentals of Heat and Mass Transfer (John Wiley \& Sons, New York, 1996); J. Niemela, L. Skrbek, K. Sreenivasan, and R. Donnelly, Nature (London) 404, 837 (2000); Y. Gasteuil, W. L. Shew, M. Gibert, F. Chillá, B. Castaing, and J. F. Pinton, Phys. Rev. Lett. 99, 234302 (2007).

[7] F. Radjai and S. Roux, Phys. Rev. Lett. 89, 064302 (2002); S. Conway, T. Shinbrot, and B. Glasser, Nature (London) 431, 433 (2004); O. Pouliquen, Phys. Rev. Lett. 93, 248001 (2004); F. Alonso-Marroquín, I. Vardoulakis, H. J. Herrmann, D. Weatherley, and P. Mora, Phys. Rev. E 74, 031306 (2006); P. Mills, P. Rognon, and F. Chevoir, Europhys. Lett. 81, 64005 (2008).

[8] D. Shi, W. Vargas, and J. McCarthy, Chem. Eng. Sci. 63, 4506 (2008); I. Figueroa, W. Vargas, and J. McCarthy, Chem. Eng. Sci. 65, 1045 (2010).

[9] C. Sammis and R. Biegel, Pure Appl. Geophys. 131, 255 (1989); J. Rice, J. Geophys. Res. 111, B05311 (2006); T. Davies and M. McSaveney, Eng. Geol. 109, 67 (2009); E. Wornyoh, V. Jasti, and C. Higgs, III, J. Tribol. 129, 438 (2007).

[10] GDR MiDi, Eur. Phys. J. E 14, 341 (2004); F. da Cruz, S. Emam, M. Prochnow, J.-N. Roux, and F. Chevoir, Phys. Rev. E 72, 021309 (2005).

[11] M. Cooper, B. Mikic, and M. Yovanovich, Int. J. Heat Mass Transf. 12, 279 (1969).

[12] J. Sun and M. Chen, Int. J. Heat Mass Transf. 31, 969 (1988).

[13] Results correspond to cell dimensions as in Fig. 1. Cells with twice the height did not show much difference. LeesEdwards boundary conditions are used with a shear rate imposed in a periodic domain. The cell contracts or dilates along $y$ according to $M \ddot{H}=\Delta \sigma_{y y}, \Delta \sigma_{y y}$ being the difference between the actual normal stress and the targeted value; we set $M=1$ (mass per unit area), leading to small normal stress fluctuations $(<1 \%)$. 